\title{
Locomotor pattern in paraplegic patients: training effects and recovery of spinal cord function
}

\author{
V Dietz, M Wirz, A Curt and G Colombo \\ Paraplegic Centre, University Hospital Balgrist, Zurich, Switzerland
}

\begin{abstract}
Recent studies have shown that a locomotor pattern can be induced and utilized by paraplegic patients under conditions of body unloading using a moving treadmill. The present study investigated the behaviour of the locomotor pattern and also the relationship of its development to the spontaneous recovery of spinal cord function assessed by clinical and electrophysiological (tibial nerve somatosensory evoked potentials and motor evoked potentials) examinations. The earliest time that spinal locomotor activity could be induced was when signs of spinal shock had disappeared. This activity was distinct from spinal stretch reflex activity. In complete paraplegic patients the locomotor pattern improved spontaneously without training. This was coincident with both an increase of gastrocnemius electromyographic activity during the stance phase of gait and a decrease of body unloading. These effects reached a plateau after about 5 weeks. In complete and incomplete paraplegic patients a near linear increase of gastrocnemius electromyographic activity occurred during the stance phase of a step cycle with daily locomotor training over the whole training period of 12 weeks. This was also coincident with a significant decrease of body unloading. In contrast to this, neither clinical nor electrophysiological examination scores improved after the onset of training in both patient groups. Only in incomplete paraplegic patients was there recovery, albeit statistically insignificant, of spinal cord function according to the sensory and motor scores obtained in the neurological examination during the time period before onset of training. An improvement of locomotor function by training was also seen in patients with paraplegia due to a cauda lesion. Such training effects on muscles and tendons could be separated from those on the spinal locomotor centres. The findings of this study may be relevant for the future clinical treatment of paraplegic patients.
\end{abstract}

Keywords: locomotor training; spinal locomotor pattern; ASIA score; tibial nerve somatosensory evoked potential; motor evoked potential

\section{Introduction}

It has been demonstrated that it is possible to induce locomotor movements with appropriate leg muscle activation in the chronic spinal cat (for review see Grillner $\left.^{1}\right)$. The pre-requisites for this to occur were partial unloading of the body and standing with the hindlimbs on a moving treadmill. Subsequently, positive effects of both locomotor training and the application of noradrenergic substances were described in this preparation. ${ }^{2}$

Similarly to the effects seen in the cat locomotor movements can be induced and be utilized by paraplegic patients with an incomplete lesion, using partial unloading of the body while standing on a moving treadmill. ${ }^{2,3}$ Indeed, recent studies in such patients have shown a significant increase in electromyographic (EMG) activity in the leg extensor muscles during training, an effect which was suggested to be

Correspondence: Prof V Dietz, Paraplegic Centre, University Hospital Balgrist, Forchstrasse 340, CH-8008 Zurich, Switzerland connected with improvement of locomotor function., ${ }^{4,5}$ Furthermore it was possible to show that even in patients who have complete paraplegia locomotor patterns could be induced ${ }^{6}$ and leg extensor EMG increased during training, ${ }^{4,5}$ although these patients did not have an improvement in their locomotor abilities.

Nevertheless, several questions remain with respect to the locomotor pattern induced in paraplegic patients. There is not yet sufficient information about the spontaneous recovery of locomotor function in these patients which may interfere with effects of locomotor training. In addition, it is still unclear to what degree the effects of locomotor training could be attributed to a training of spinal locomotor centres, as locomotor training will also affect leg muscles and tendons resulting in an improvement of locomotor performance.

Therefore, the aim of this study was to elucidate and define more precisely the function and behaviour of the spinal locomotor apparatus in humans. Only 
systematic assessment of leg muscle EMG activity during the course of the training can reflect the neuronal activity of the spinal cord and can indirectly provide evidence for training effects on the spinal pattern generator. The effects of locomotor training were related to clinical and electrophysiological examinations over the time course of rehabilitation. The present studies were extended also for paraplegic patients with cauda lesions in order to assess the effects of the training programme on the tendons and muscles of the legs. Furthermore, we have analyzed the spontaneous recovery of locomotor function in paraplegic patients. The observations made here may serve as a basis for future pharmacological and electrophysiological manipulation of the locomotor pattern generator in order to further improve mobility in paraplegic patients.

\section{Materials and methods}

\section{General procedures and patients}

The local ethical committee granted permission to make recordings during treadmill locomotion on patients with complete and incomplete paraplegia. Daily locomotor training and weekly recordings were done in five patients with complete para-/tetraplegia (mean age 33), seven patients with incomplete para-/ tetraplegia (mean age 31 ), two patients with incomplete paraplegia due to a cauda lesion (mean age 34) and three age-matched normal subjects. The clinical diagnosis of a complete spinal cord lesion was supplemented by electrophysiological and radiological (eg magnetic resonance imaging) data. Patients with incomplete paraplegia were unable to perform stepping movements during the first trials (for further details see Table 1). Most patients were spastic and exhibited exaggerated tendon tap reflexes in the legs when locomotor training was started. However, in a few (three) patients, recordings of locomotor activity were already taken at an earlier stage during spinal shock when tendon tap reflexes were still abolished. For some specific questions, recordings were made also in patients not listed in Table 1 .

All patients underwent daily locomotor training on the treadmill (approximately $300 \mathrm{~m}$ of walking) starting from 49 to 323 days post-trauma (mean 125 days). In completely paraplegic patients and at the beginning of the training in all incompletely paraplegic patients, stepping movements could easiest be induced with the treadmill moving at a speed of approximately $1.5 \mathrm{~km} / \mathrm{h}$. With partial unloading of body weight (up to $80 \%$ of the weight was unloaded), physiotherapists assisted the movements of the legs on both sides, especially around knee extension and during transitions from stance to swing and vice versa of the respective leg. Unloading of the patients was achieved by suspending them from a parachute harness connected to an overhead crane. The degree of unloading during one training session was provided by a scale on the crane.

Standardized neurological examinations of the patients were performed according to the American Spinal Injury Association (ASIA) protocol. ${ }^{7}$ The ASIA motor (maximum 100 points) and sensory (maximum for both aesthesia and algesia 112 points) scores indicated the severity of the spinal cord injury (SCI) lesion.

\section{Electrophysiological recordings}

Electromyographic (EMG) recordings were made weekly during treadmill locomotion using surface electrodes $(5 \mathrm{~cm}$ apart) from the medial gastrocnemius

Table 1 Patients with incomplete and complete paraplegia who participated in the locomotor training programme

\begin{tabular}{|c|c|c|c|c|c|}
\hline Patient \# & $\begin{array}{c}\text { Age } \\
\text { [years] }\end{array}$ & $\operatorname{Sex}$ & $\begin{array}{l}\text { Level of } \\
\text { lesion }\end{array}$ & $\begin{array}{c}\text { ASIA } \\
\text { impairment } \\
\text { scale }\end{array}$ & Drugs \\
\hline \multicolumn{6}{|c|}{ Incomplete paraplegia } \\
\hline 1 & 28 & M & C5 & $\mathrm{C}$ & Clonazepam \\
\hline 2 & 73 & M & $\mathrm{C} 4$ & $\mathrm{D}$ & \\
\hline 3 & 21 & M & $\mathrm{C} 5$ & $\mathrm{D}$ & \\
\hline 4 & 21 & M & T7 & $\mathrm{C} / \mathrm{D}$ & Baclofen \\
\hline 5 & 50 & M & C5 & $\mathrm{C}$ & \\
\hline 6 & 13 & M & C6 & $\mathrm{D}$ & Clonazepam/Baclofen \\
\hline 7 & 14 & M & $\mathrm{C} 5$ & $\mathrm{C}$ & Baclofen \\
\hline \multicolumn{6}{|c|}{ Complete paraplegia } \\
\hline 8 & 53 & M & T7 & B & Clonazepam \\
\hline 9 & 22 & M & T5 & A & \\
\hline 10 & 21 & $\mathrm{~F}$ & C7 & A & Clonazepam \\
\hline 11 & 46 & M & $\mathrm{C} 5 / \mathrm{C} 6$ & A & \\
\hline 12 & 22 & M & C6 & A & Clonazepam \\
\hline \multicolumn{6}{|c|}{ Incomplete cauda } \\
\hline 13 & 34 & M & L2 & B & \\
\hline 14 & 33 & M & L5 & B & \\
\hline
\end{tabular}


(GM), and tibialis anterior (TA) muscles of both legs. Ankles and knee-joint movements were monitored using mechanical goniometers fixed at the lateral aspect of the right and left ankles and knees. A force plate signal underneath the treadmill belt provided a record of the vertical force exerted on the treadmill. This signal indicated onset of the step cycle.

Somatosensory evoked potentials (SSEP) were elicited by electrical stimulation $(0.2 \mathrm{~ms}$ duration, square wave, applied at $3 \mathrm{~Hz}$ ) of the tibial nerves delivered at the medial aspect of the ankles of both legs (for details see Curt and Dietz ${ }^{8}$ ). The cathode was placed 2 to $3 \mathrm{~cm}$ proximal to the anode. Subjects were lying prone at ambient room temperature. The stimulus intensity was adjusted to produce a clear muscle contraction. The recording electrodes (conventional $0.5 \mathrm{~cm}$ gold cup electrodes) were attached to the skin over the first lumbar vertebra, L1 (to control the effectiveness of electrical stimulation and transmission of the potentials along the peripheral nerve segments). Scalp electrodes were positioned at $\mathrm{Cz}-\mathrm{Fz}$ (International 10/20 electrode system). The electrode impedance was maintained at less $5 \mathrm{k} \Omega$. The amplifier was set at $5 \mu \mathrm{V} / \mathrm{div}$ and the time of analysis at $100 \mathrm{~ms}$. Two sets of 500 responses were averaged and superimposed to ensure consistency.

The N 40 latencies and amplitudes of tibial SSEP recordings were determined and the results were classified into five categories according to Kovindha and Mahachai. ${ }^{9}$ This classification reflects different degrees of impaired impulse transmission within the spinal cord (category No 1: No cortical SSEP recording; (2) Pathological latency and amplitude of the N 40 component; (3) Delayed latency with normal amplitude of the N 40 component; (4) Normal latency with reduced amplitude of the $\mathrm{N} 40$ component; (5) Normal latency and amplitude of the $\mathrm{N} 40$ component). The limits to define significant pathological results were defined according to values reported elsewhere. ${ }^{10,11}$

The patients were examined monthly during the first 3 months post-trauma and again 6 months posttrauma. The first examination was done on the first or second day after hospitalisation, but usually within the first 2 weeks post-trauma.

Motor evoked potentials (MEP) due to transcranial magnetic stimulation were evoked using a commercially available stimulator with a flat round coil (Mag PRO, DANTEC) localized over $\mathrm{Cz}$ (for details see Curt and Dietz ${ }^{12}$ ). The stimulus waveform was biphasic, the pulse-width was $200 \mu \mathrm{s}$ with an intensity of $100-160 \mathrm{~A} / \mu \mathrm{s}$. Current direction was clockwise. With the patient in a supine position, the stimulus intensity was gradually increased until a maximal muscle response was obtained in the TA muscle. The latencies and amplitudes of TA EMG responses were determined and the results were classified into the five categories described above for the SSEP recordings. The limits to define significant pathological results were as previously described. ${ }^{13}$
The examinations were performed together with the SSEP-recordings.

\section{Data analysis}

EMG recordings were amplified (microvolt amplifier; bandpass filter, $30-300 \mathrm{~Hz}$ ) and were transferred together with the biomechanical signals to a PCmicrocomputer systems via an analog-to-digital converter. All signals were sampled at $600 \mathrm{~Hz}$ and, after rectification of the EMG, were averaged over 20 step cycles. The force signal from the right foot fall and onset of right stance phase was used as a trigger for averaging the EMG and biomechanical signals. Individual step cycles were normalized to a relative time scale of one step cycle starting and ending with the right heel strike. For a detailed description of the recording techniques and signal analysis, see earlier articles. $^{4,14}$

To investigate changes in gastrocnemius EMG activity as a function of time and of training, the signal energy (RMS = root mean square) was determined during a $20 \%$ interval of the step cycle ending $5 \%$ before the end of the stance phase of the step cycle, a period in which gastrocnemius activation was expected to occur (Figure 1). By this procedure only changes in amplitude but not in timing of EMG activity were assessed. The knee joint signal (mid of flexion movement) was used to trigger the end of the stance phase, because this signal was most consistent between the different patient groups.

To allow intersubject comparison of changes in gastrocnemius EMG activity as a function of training within 12 weeks, the individual RMS amplitudes were normalized. This was done by rank order of the highest to lowest values within the 12 weeks of training.

\section{Statistics}

A SAS-package (information delivery system) was used. Time course changes of repeated measurements from a data collection session on the same individuals were investigated using the Spearman correlation test. The coefficients were calculated for the clinical (ASIA motor and sensory) scores, the SSEP/MEP values and the rank orders of RMS EMG amplitudes over the time after injury and of the training programme respectively. For the separation of the two effects, body (un)loading and training, on the time course of changes in EMG amplitude (rank orders) the Spearman partial correlation coefficients were calculated. The level of significance was set at $P<0.05$.

\section{Results}

In a first part data will be presented which may influence the leg muscle activation during the locomotor training. In a second part the changes of biomechanical and EMG parameters obtained during 
the training period will be related to electrophysiological and clinical parameters indicating a possible spontaneous recovery of spinal cord function.
Effect of locomotor training

In this section the leg muscle activation pattern at the beginning and the end of a locomotor training in both
Gastrocnemius medialis

\section{A. Complete SCI}
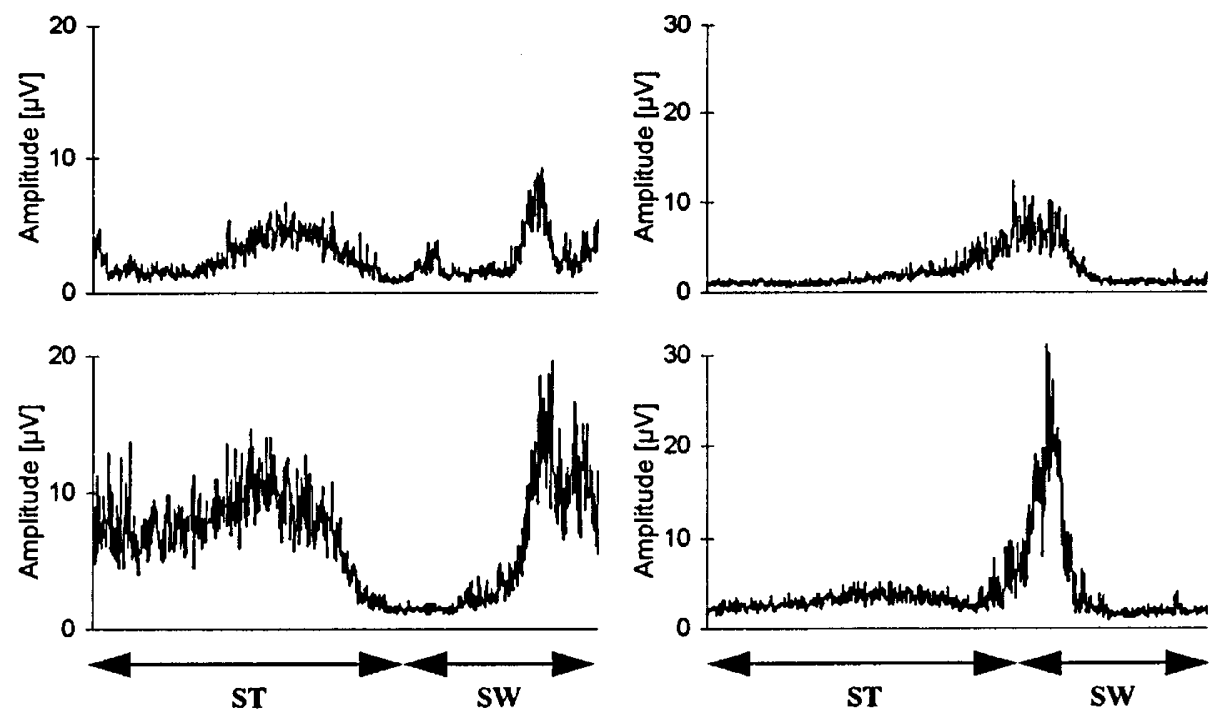

12 weeks

of training

\section{B. Incomplete SCI}
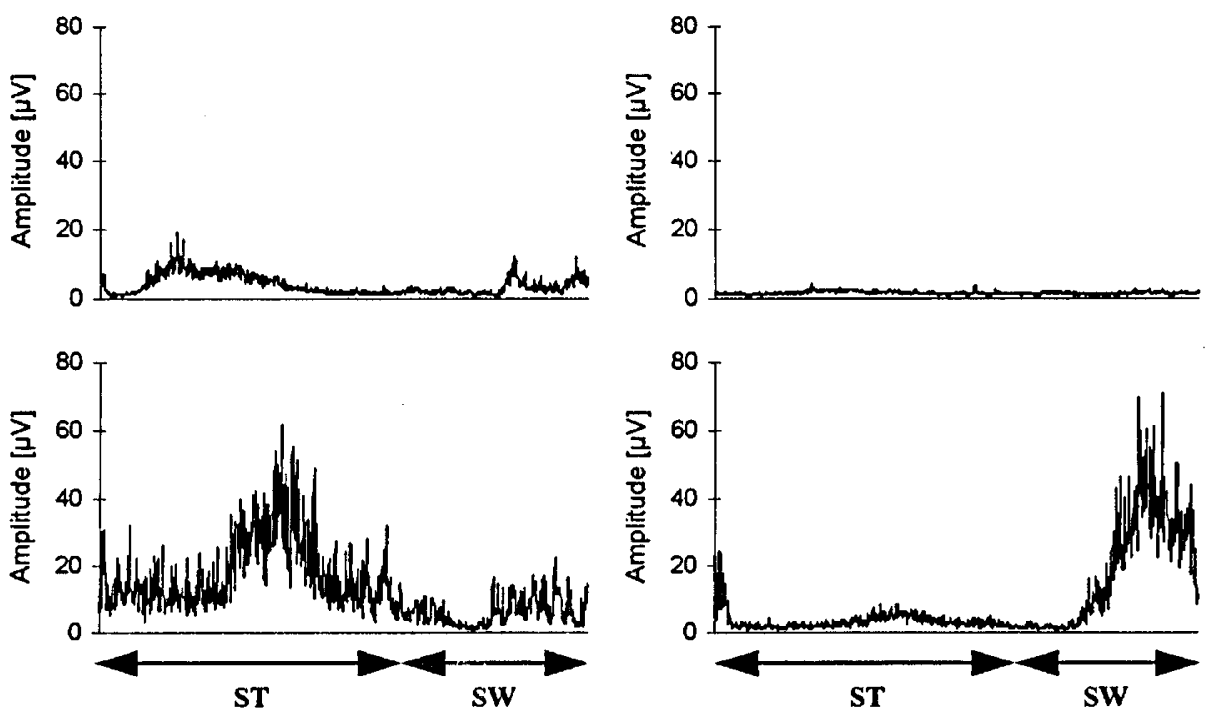

begin training

12 weeks

of training

Figure 1 Lower leg muscle electromyographic (EMG) activity and joint movements of (a) a completely and (b) an incompletely paraplegic patient at the beginning of and 12 weeks after daily locomotor training. The unloading amounted to $75 \%$ (beginning) and $50 \%$ (end) of body weight for the completely, and $80 \%$ (beginning) and $45 \%$ (end) of body weight for the incompletely paraplegic patient. Note the different calibrations of EMG amplitude 
completely and incompletely paraplegic patients will be presented.

Figure 1 shows typical training effects (over 12 weeks) on the lower leg muscle EMG-activity in a patient with complete paraplegia (Figure 1a; no 10, Table 1) and a patient with incomplete paraplegia (Figure 1b, no 6, Table 1). It is clear that in both cases the gastrocnemius (GM) and tibialis anterior (TA) EMG activity increased mainly during the stance and swing phases, respectively, ie the appropriate phases of the step cycle. However the changes in the complete paraplegic patient were less dramatic (see different calibration in Figure 1a and b). Therefore, locomotor movements in the complete paraplegic patients still had to be assisted externally at the end of the training period. However unloading could be decreased from $65 \%$ to $50 \%$ of body weight. In contrast, the incompletely paraplegic patient was able to walk with some support on normal ground conditions after the training period.

\section{Influence of stretch reflex activity and body load}

Two factors may influence the locomotor pattern during training besides the spontaneous recovery of spinal cord function: stretch reflex activity and actual body load.

The first factor concerns the origin of the gastrocnemius EMG activity recorded during treadmill locomotion and its relationship to stretch reflexes. A locomotor activity could only be recorded after spinal shock disappeared, ie with the occurrence of exaggerated tendon reflexes. Therefore, it may reflect the activity of stretch reflexes. In Figure 2 the amplitude of the GM and TA EMG activity during the step cycle was plotted against the lengthening and shortening phases of the respective muscle in a healthy subject and a complete paraplegic patient (no 11, Table 1) 26 weeks after injury. For the GM it was taken into account, that this is a biarticulate muscle. In both the healthy subject and the paraplegic patient there was a near equal distribution of EMG activity during muscle lengthening and shortening in these muscles. An about equal distribution of EMG-activity was also found in all other patients. However, as shown before the amplitude of EMG activity was considerably smaller in the patient than in the healthy subject.

The other factor concerns the effect of actual body load on the leg extensor EMG. The amount of
Patient with complete paraplegia
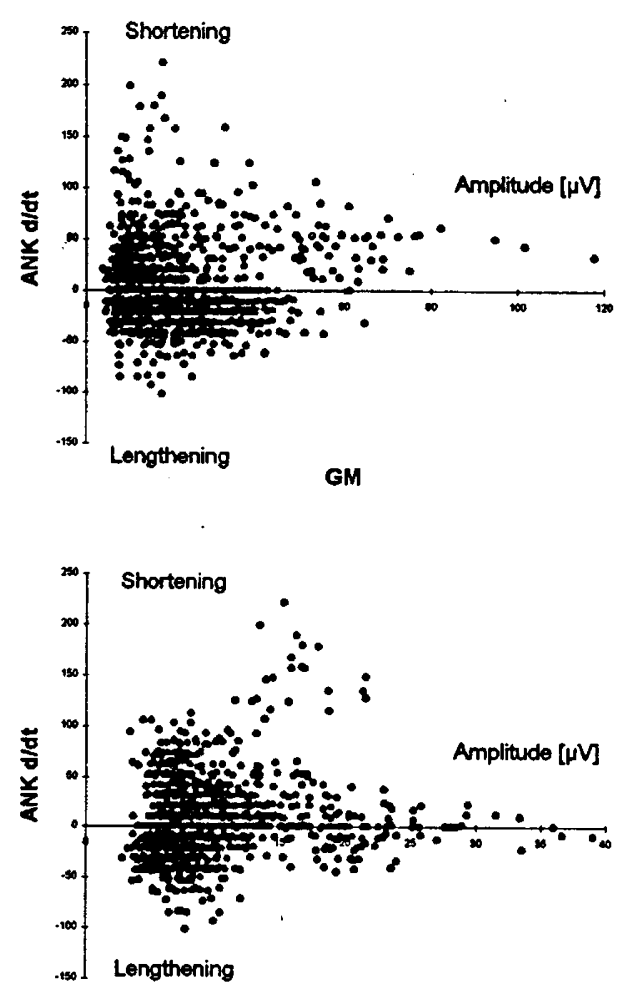

TA
Healthy subject
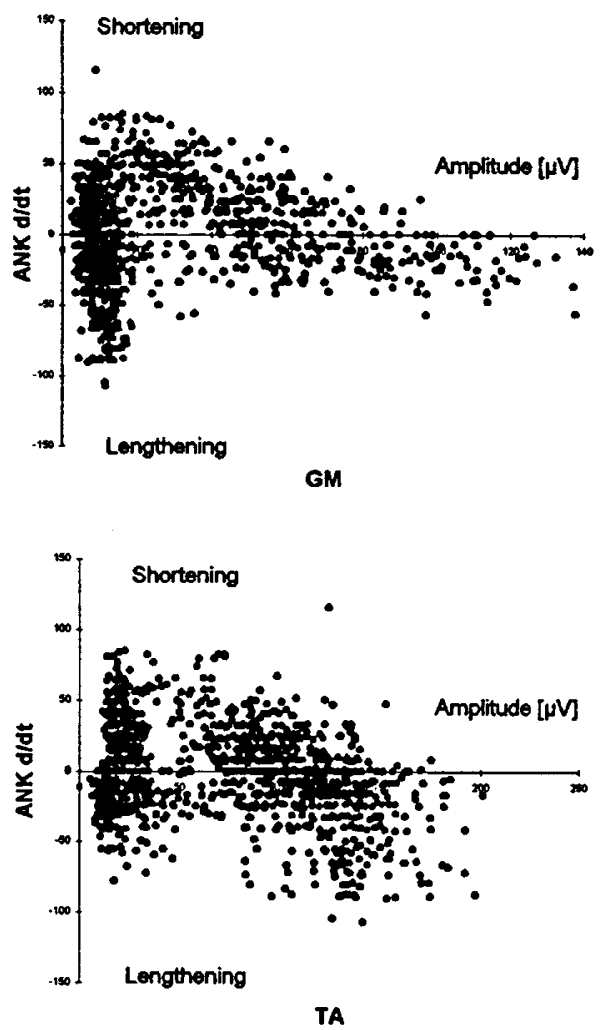

Figure 2 Relationship between muscle lengthening and shortening (velocity at the ankle, ANK d/dt) and EMG amplitude in the gastrocnemius and tibialis anterior muscles of a completely paraplegic patient and a healthy subject within a step cycle. One point represents the activity level expressed as RMS sampled at $600 \mathrm{~Hz}$ over an averaged $(n=20)$ step cycle 
unloading and re-loading of the body obviously played a major role for successful locomotor training. Unloading of the body was required in order to allow the induction of locomotor movements with an adequate support of the body by the feet during the stance phase. Therefore, the effect of different amounts of unloading on the locomotor activity was studied in an incompletely (motor function completely) paraplegic patient (no 8, Table 1) and a healthy subject. It became obvious that the absolute range of change in GM-EMG amplitude at various loads was similar in the patient and the healthy subject. However, the activity level at which this change took place was much lower (by a factor of 4 ) in the patient. In addition the shape of GMEMG activity was different with a broader GM activation over the stance phase in the patient. During the normal locomotor training program of this patient, body unloading was set at about $40 \%$, which allowed the physiotherapists to induce stepping movements with only slight external assistance. When the body was unloaded only by $13 \%$, a greater effort was required by the external assistant in order to avoid knee flexion during the stance phase.

\section{Locomotor activity and spinal cord function: complete} paraplegic patients

In the following the question is followed in how far a real training effect on the locomotor pattern can be evaluated: To what degree could the changes of the locomotor pattern seen in paraplegic patients during the training period be attributed to a spontaneous recovery following a spinal lesion?

In a completely paraplegic patient (no 9, Table 1) gastrocnemius EMG recordings were taken each week during the locomotor cycle (with external assistance) without a daily training. The recordings were made in a time period (8th-20th week post-injury) during which the locomotor training was usually performed in the other patients. It became obvious that during the first recordings no visible EMG activity was present. At this time also tendon tap reflexes at the legs were still abolished. After spinal shock (when tendon tap reflexes reappeared) the locomotor pattern became spontaneously established (although induced by external manipulations of the legs). The increase in GM-EMG activity took place over about 4 weeks without any noticeable change in the timing of the EMG-pattern. The increase of GM activity was restricted to the stance phase of the step cycle. After this time period a plateau was reached without further significant changes in EMG activity. Two further patients (not listed in Table 1) showed similar behaviour, reaching a plateau after 4 to 6 weeks (from the beginning of the recordings on).

Figure 3 and Table 2 summarise the results of all five trained complete paraplegic patients. In Table 2 the absolute individual values of GM-EMG changes over time are displayed. To make a comparison possible between patients, the quantified gastrocnemius EMG values were normalized by rank order from highest to lowest values obtained during the training period of 12 weeks (recordings were performed weekly). Unloading is displayed in percent of body weight. There was a significant increase of GM-EMG activity and decrease of body unloading during training. The degree of unloading was adjusted to the range where an optimal performance of locomotor movements was still possible. The increase of GM-EMG activity achieved during the training period was mainly restricted to the stance phase of the step cycle (not shown here). Neither ASIA motor and sensory scores (the scores were related to the function above the level of lesion) nor the scores of tibial nerve SSEP (all were absent) or transcranial magnetic stimulation (no potential could be evoked in any patient) changed significantly during the observation period, ie before and after onset of training (not shown here). The increase of GM-EMG activity might be attributed more or less exclusively to the decrease of body unloading during training. However, when the effect of (un)loading was separated by the Spearman partial correlation test (see Methods), the effect of training was still significant (coefficient 0.369; $P<0.05)$

Locomotor activity and spinal cord function: incomplete paraplegic patients

As before, the effects of the locomotor training for the incomplete paraplegic patient group may be due to the

\section{Complete SCI}

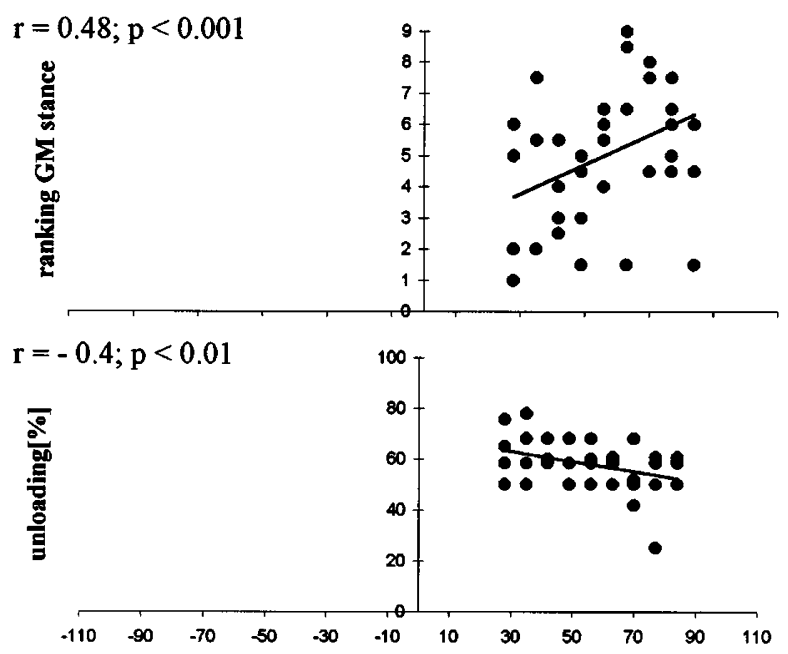

Figure 3 Relative values of gastrocnemius EMG activity during the stance phase (after normalization of the individual data, see Materials and methods) and percentage of body unloading during the time course of daily locomotor training. The data of all five completely paraplegic patients are shown 
Table 2 Individual changes of quantified GM-EMG activity (slope of linear regression) in the stance phase of the step cycle during the course of a daily locomotor training ( $r=$ correlation coefficient; $P=$ significance level)

\begin{tabular}{|c|c|c|c|c|c|c|c|}
\hline \multirow[b]{2}{*}{ Patient \# } & \multirow[b]{2}{*}{ Measurements } & \multicolumn{3}{|c|}{ Right leg } & \multicolumn{3}{|c|}{ Left leg } \\
\hline & & $r$ & Slope & $p$ & $r$ & Slope & $p$ \\
\hline \multicolumn{8}{|c|}{ Incomplete paraplegia } \\
\hline 1 & 10 & 0.87 & $3.10 \mathrm{E}-07$ & 0.001 & 0.88 & 2.24E-07 & 0.001 \\
\hline 2 & 24 & 0.12 & $4.82 \mathrm{E}-08$ & 0.589 & 0.06 & $1.28 \mathrm{E}-08$ & 0.773 \\
\hline 3 & 26 & 0.59 & $2.19 \mathrm{E}-07$ & 0.001 & 0.62 & $8.11 \mathrm{E}-08$ & 0.001 \\
\hline 4 & 23 & 0.65 & $8.68 \mathrm{E}-08$ & 0.001 & -0.19 & $-8.03 \mathrm{E}-09$ & 0.375 \\
\hline 5 & 17 & 0.07 & $5.42 \mathrm{E}-09$ & 0.785 & 0.32 & 3.24E-08 & 0.208 \\
\hline 6 & 18 & 0.4 & $1.38 \mathrm{E}-07$ & 0.101 & 0.34 & $1.08 \mathrm{E}-07$ & 0.171 \\
\hline 7 & 19 & 0.68 & $1.88 \mathrm{E}-07$ & 0.001 & 0.2 & 3.37E-08 & 0.422 \\
\hline \multicolumn{8}{|c|}{ Complete paraplegia } \\
\hline 8 & 14 & 0.59 & $8.50 \mathrm{E}-08$ & 0.028 & 0.5 & $6.64 \mathrm{E}-08$ & 0.068 \\
\hline 9 & 12 & 0.15 & $3.03 \mathrm{E}-08$ & 0.642 & 0.04 & 8.88E-09 & 0.901 \\
\hline 10 & 19 & 0.49 & $1.24 \mathrm{E}-07$ & 0.032 & 0.33 & 5.52E-08 & 0.173 \\
\hline 11 & 34 & -0.43 & $-6.07 \mathrm{E}-08$ & 0.012 & -0.11 & $-5.69 \mathrm{E}-09$ & 0.533 \\
\hline 12 & 8 & 0.42 & 2.50E-08 & 0.296 & 0.62 & 3.68E-08 & 0.098 \\
\hline \multicolumn{8}{|c|}{ Incomplete cauda } \\
\hline 13 & 11 & -0.13 & $-3.86 \mathrm{E}-09$ & 0.698 & 0.11 & 9.23E-09 & 0.752 \\
\hline 14 & 14 & -0.25 & $-3.13 \mathrm{E}-08$ & 0.382 & -0.86 & $-1.44 \mathrm{E}-07$ & 0.001 \\
\hline
\end{tabular}

spontaneous recovery of spinal cord function which usually takes place $2-3$ months following spinal cord injury. $^{7}$ To assess this spontaneous recovery, the training effects of locomotor function were related to clinical and electrophysiological examinations. Figure 4 summarizes the results obtained in all seven incomplete paraplegic patients. There was a significant increase in gastrocnemius EMG activity during the stance phase of gait and a corresponding decrease of body unloading during the training period. Also in this patient group the training effect on the GM-EMG was still significant (coefficient $0.578 ; P<0.001$ ) after extraction of the (un)loading effect (see Methods). Only, before the onset of locomotor training there was a visible increase of sensory and motor ASIA scores. Even when the data of this period were calculated separately, the increase was just below the limit of significance $(P=0.06)$. During the time period following onset of training, there was no further increase in the ASIA scores. The electrophysiological examinations (tibial nerve SSEP and MEP) neither before nor after onset of training showed any positive or negative trends.

\section{Incomplete cauda lesions}

Figure 5 shows a typical example of a patient with a cauda lesion (no 13, Table 1), before and after a 12 week training period. From a clinical point of view this patient profited from the locomotor training. At the beginning of training, locomotor movements could only be induced and be trained with external assistance when body was considerably unloaded (30 of $65 \mathrm{~kg}$ ). At the end of training the unloading was only 20 of $65 \mathrm{~kg}$ and the patient was able to perform stepping movements on normal ground conditions with minimal support (crutches). However, EMG activity in upper and lower leg muscles was little changed. While there was almost no EMG activity in the lower leg muscles before and after training, the amplitude of EMG activity in upper leg muscles tended to be rather smaller at the end of the training period. In two patients the effect of locomotor training was related to the clinical and electrophysiological examinations. There was a general decrease of GM-EMG activity and of body unloading during the training period although these changes were not significant. Neither clinical (sensory and motor ASIA scores) nor electrophysiological (tibial nerve SSEP, MEP scores) showed a significant change during the time periods before and after onset of training (not shown here).

\section{Discussion}

The aim of this study was to evaluate the effects of locomotor training in paraplegic patients. The main results obtained were: (1) The effects of locomotor training can be separated from the spontaneous recovery of spinal cord function in patients with SCI; (2) There is a limited contribution of stretch reflex activity to the locomotor pattern; and (3) There are non-specific effects of locomotor training on muscles and tendons. These points will be discussed with respect to their clinical significance.

\section{Indications for a spinal locomotor pattern generator}

Although there is strong evidence for a spinal locomotor generator in many mammals (for review see Grillner ${ }^{1}$, Barbeau and Rossignol ${ }^{3}$ ), its existence has, until recently, been questioned in man. ${ }^{15,16}$ Evidence for the human spinal pattern generator was 


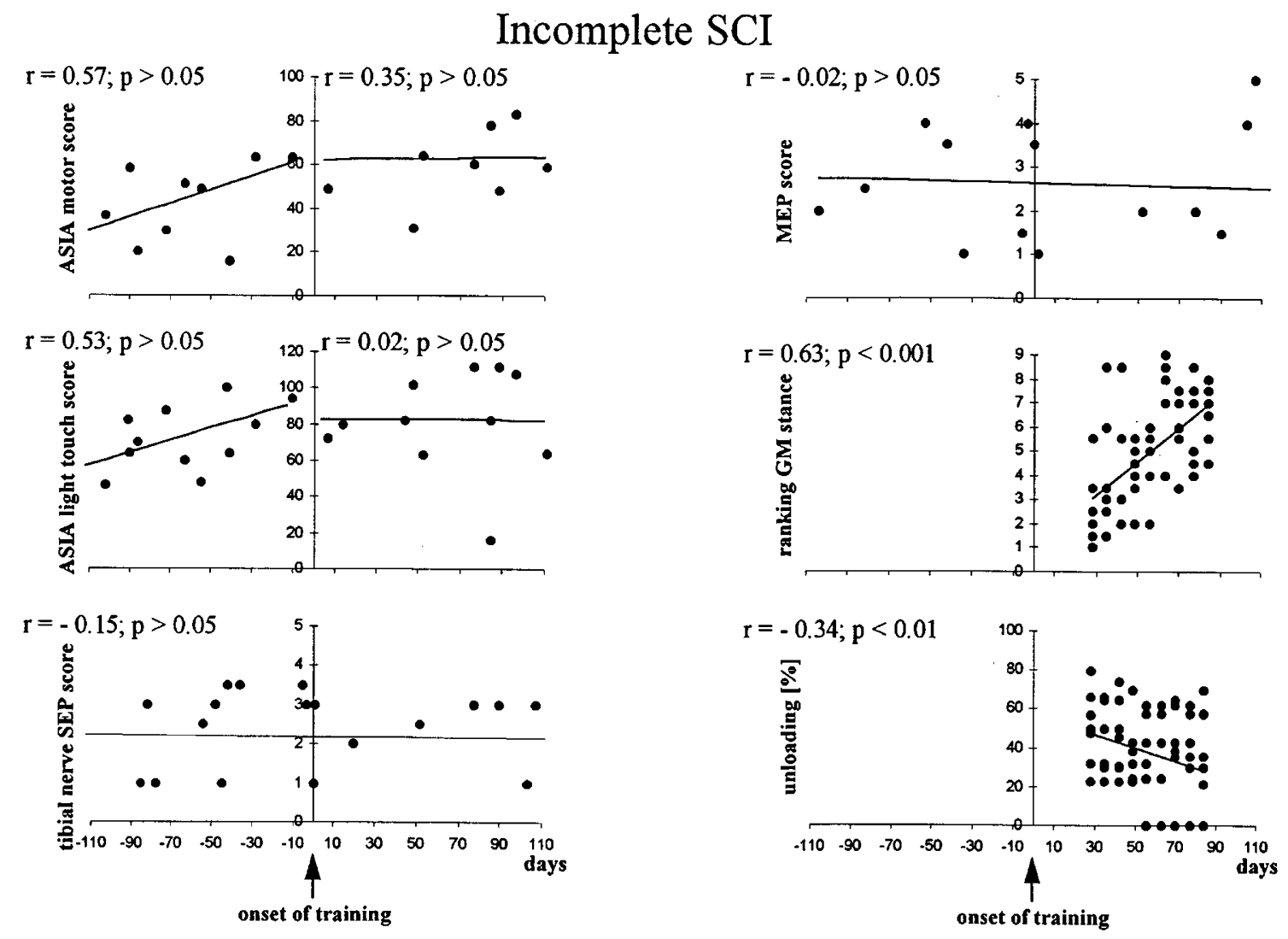

Figure 4 Clinical (ASIA motor and sensory scores) and electrophysiological (tibial nerve-SSEP and MEP scores) data as well as values of GM-EMG activity and body unloading of all seven incompletely paraplegic patients. Clinical and electrophysiological examinations were performed in each patient at least twice. For the electrophysiological recordings not all patients are represented with the same number of recordings. For further details see Figure 3

suggested by spontaneously occurring step-like movements ${ }^{17}$ and myoclonus, ${ }^{18}$ as well as from late flexion reflexes ${ }^{19}$ and from locomotor movements induced on a treadmill with body support in paraplegic patients, ${ }^{4,5}$ (for review see Barbeau and Rossignol ${ }^{3}$ ). Recent studies showed that a locomotor pattern can be induced in complete paraplegic patients when leg movements were assisted from externally. ${ }^{4-6}$ Nevertheless, the amplitude of leg muscle EMG activity in these patients was small compared to healthy subjects, (most probably due to the loss of noradrenergic influences from brainstem centres) such that no leg movements resulted from this leg muscle activation.

This study has demonstrated that a locomotor pattern can only be induced some weeks after SCI, when spinal reflexes re-appear. The question, therefore, was to what degree the EMG pattern recorded may be due to the activity of spinal stretch reflexes as suggested elsewhere. ${ }^{20}$ Correlation studies between leg muscle EMG activity and the lengthening and shortening phases of respective leg muscles showed that leg muscle EMG activity is equally distributed during the lengthening and shortening phases of the respective leg muscles. Obviously, following spinal shock after a SCI, a recovery of several distinct spinal cord functions takes place.

\section{Locomotor training effects}

The beneficial effect of locomotor training for incomplete paraplegic patients is well established ${ }^{21,22}$ (for review see Barbeau and Rossignal ${ }^{3}$ ) and recent investigations show that patients who undergo locomotor training have greater mobility compared to a control group without training. ${ }^{23}$ Nevertheless, it remained unclear to what extent these training effects are due to a training of spinal locomotor centres. Only by systematic recordings of leg muscle EMG, reflecting the activity of spinal neuronal circuits, the effect of this presumed 'spinal locomotor pattern generator' can be separated from rather non-specific effects on muscles and tendons. The present observations on patients with paraplegia due to a cauda, ie peripheral nerve lesion, show that a locomotor training indeed results in an improvement of locomotor function which is not connected with a corresponding change in leg muscle 
EMG activity. Therefore, the improvements of locomotor function described earlier for $\mathrm{cat}^{2}$ and $\operatorname{man}^{3,22}$ can at least be attributed partially to nonspecific effects on the locomotor apparatus, ie muscular tendon-systems.
Body unloading is obviously of crucial importance to induce training effects on the locomotor centres. The range of body unloading that allows stepping movements and optimal activation of leg muscles for body support by the legs during the stance phase is

\section{Patient with incomplete cauda lesion}

Begin training (Unloading $45 \mathrm{~kg}$ )
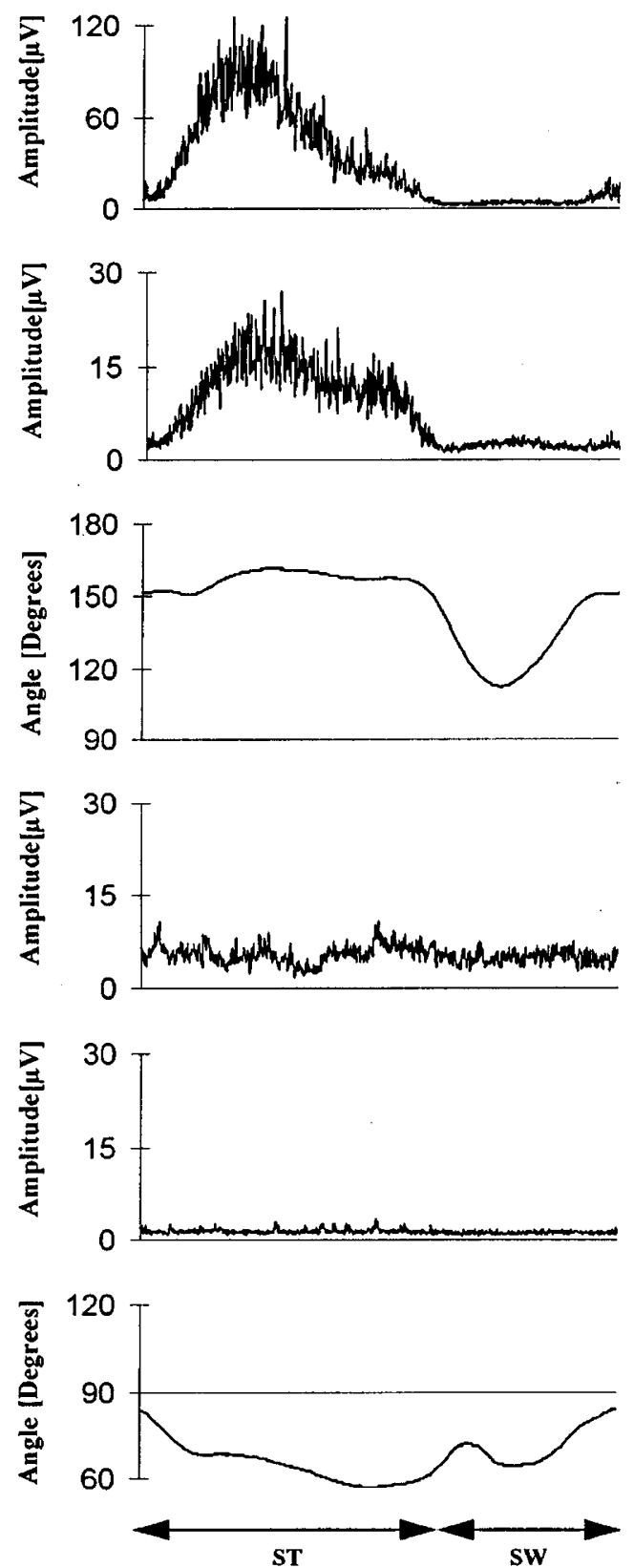

End training (Unloading $20 \mathrm{~kg}$ )
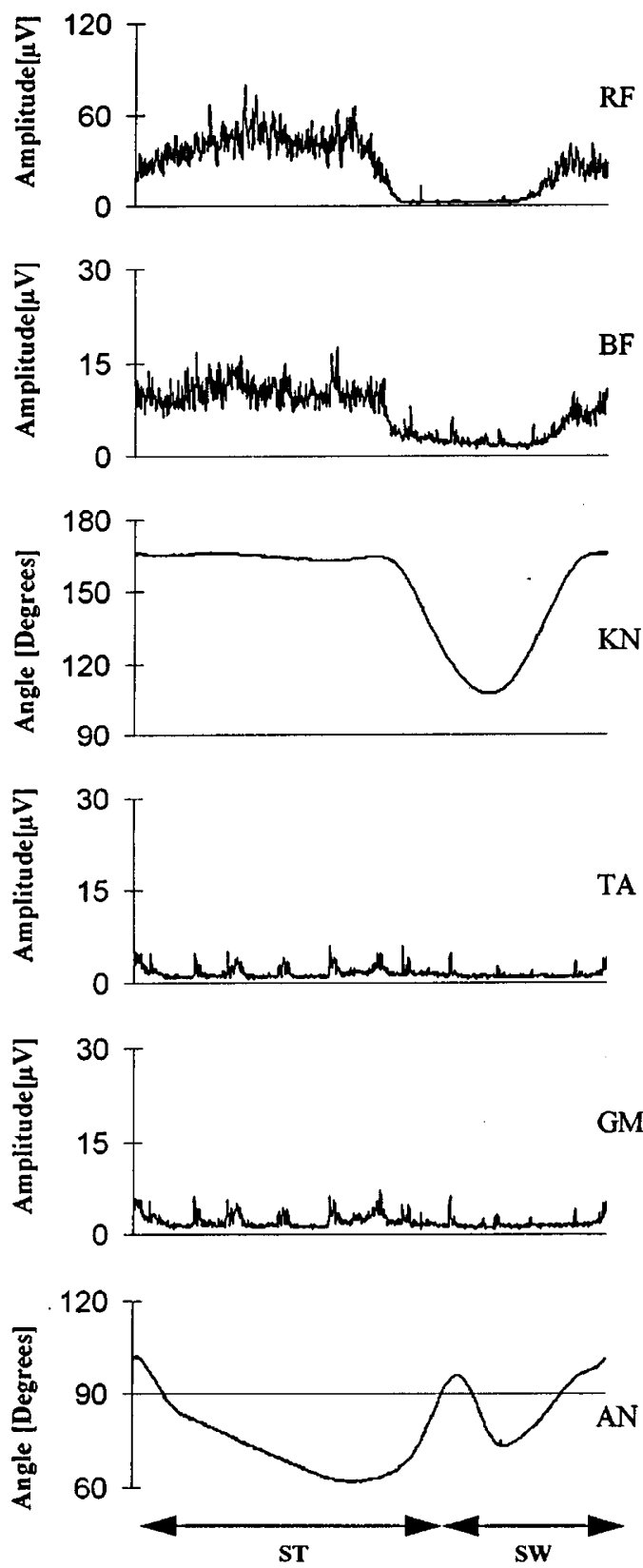

Figure 5 EMG activity and joint movements of an incompletely paraplegic patient due to cauda lesion before (left side) and after (right side) a training period of 10 weeks. For further details see Figure 1 
limited in paraplegic patients. Obviously, afferent input from receptors signaling contact forces during the stance phase is essential for the activation of spinal locomotor centres ${ }^{24}$ and therefore for the training effects described here. Although the amplitude of leg extensor EMG in both paraplegic and healthy subjects depended upon the actual body load during the stepping movements, the absolute level of EMG activity was consideraby lower in the patient than in the healthy subject. This makes the body unloading necessary for the locomotor training. There is, indeed, increasing evidence in $\mathrm{cat}^{25}$ and $\operatorname{man}^{26}$ for a contribution of load receptors to the activation of leg extensor during stance and locomotion.

\section{Spontaneous recovery of locomotor function}

Recent studies have shown that during locomotor training gastrocnemius EMG activity increased during the stance phase even in complete paraplegic patients. Nevertheless, from the latter study, this improvement of locomotor activity could have been attributed to spontaneous recovery of spinal cord function resulting in the observed improvement in clinical and electrophysiological examinations of spinal cord function. It is well known that recovery of spinal cord function can occur over several months following SCI. ${ }^{27,28}$ From the present observations in both, incomplete and complete paraplegic patients, there can be little doubt that the increase of leg extensor EMG activity, connected with a decrease of body unloading, occurs independently of the recovery of spinal cord function assessed by clinical and electrophysiological means. Of course, clinical and electrophysiological tools are too crude to rule out recovery of any spinal cord function. Nevertheless, locomotor training obviously plays an important role for the effects on leg extensor muscle EMG.

In complete paraplegic patients spontaneous development of a locomotor pattern could be observed. However, this occurred after spinal shock had disappeared and reached a plateau usually about 4 weeks later. In contrast, under locomotor training which usually started at about the same time after spinal shock, GM-EMG activity further increased during the stance phase, ie after the time when a plateau had been expected to be reached spontaneously. This effect was connected with progressive loading (ie reduced unloading) during locomotion.

In addition, one might argue that the increase of GM-EMG during the course of training is mainly due to the decrease of body unloading. However, after statistical separation of the (un)loading effect, there remained a significant effect of training. Therefore, one may conclude that there are specific training effects on spinal locomotor centres which can lead to an improvement of locomotor function in paraplegic patients.

Looking ahead it may be important to discover new means to further enhance the amplitude of leg extensor EMG activity in complete or almost complete paraplegic patients in order to achieve an even greater mobility in such patients. This may be achieved by the application of new noradrenergic drugs which have a more selective action on the EMG activity compared to the ones already applied (clonidine and epinephrine $^{4}$ ). The most promising approach may, however, be to induce some regeneration of corticospinal axons within the spinal cord. Recent experiments in rats ${ }^{29}$ indicate that this goal may be achieved during the next few years.

\section{Acknowledgements}

This work was supported by grants from the 'Swiss National Science Foundation' (no 31-42899.95) and the 'Schweizerische Bankgesellschaft' on behalf of a client. We thank Dr I Gibson for correcting the English text and Th. Erni for statistical support.

\section{References}

1 Grillner S. Control of locomotion in bipeds, tetrapods, and fish. In: Brookhart M, Mountcastle VB (eds), Handbook of Physiology. The Nervous System vol II, Motor Control, part 2. American Physiological Society, Washington, DC, 1981, pp 1179-1235.

2 Barbeau H, Rossignol S. Recovery of locomotion after chronic spinalization in the adult cat. Brain Res 1987; 412: 84-95.

3 Barbeau H, Rossignol S. Enhancement of locomotor recovery following spinal cord injury. Curr Opinion Neurol [Review] 1994; 7: $517-524$.

4 Dietz V, Colombo G, Jensen L, Baumgartner L. Locomotor capacity of spinal cord in paraplegic patients. Ann Neurol 1995; 37: $574-582$.

5 Dietz V, Colombo G, Jensen L. Locomotor activity in spinal man. The Lancet 1994; 344: 1260-1263.

6 Dobkin BH, Harkema S, Requejo P, Edgerton VR. Modulation of locomotor-like EMG activity in subjects with complete and incomplete spinal cord injury. J Neuro Rehab 1994; 9: 183-190.

7 Ditunno JF, Young W, Donovan WH, Creasey G. The International Standards Booklet for neurological and functional classification of spinal cord injury. Paraplegia 1994; 32: $70-80$.

8 Curt A, Dietz V. Ambulatory capacity in spinal cord injury: Significance of ASIA protocol and SSEP recordings in predicting outcome. Arch Phys Med Rehabil 1997; 78: 39-43.

9 Kovindha A, Mahachai R. Short-latency somatosensory evoked potentials (SSEP) of tibial nerves in spinal cord injuries. Paraplegia 1992; 30: 502-506.

10 Nuwer MR et al. IFCN recommended standards for short latency somatosensory evoked potentials. Report of an IFCN Committee. Electroenceph Clin Neurophysiol 1994; 91: 6-11.

11 Yiannikas C, Shahani BT, Young RR. Short-latency somatosensory-evoked potentials from radial, median, ulnar, and peroneal nerve stimulation in the assessment of cervical spondylosis. Arch Neurol 1986; 43: $1264-1271$.

12 Curt A, Dietz V. Functional outcome following spinal cord injury: Significance of motor evoked potentials and ASIA scores. Arch Phys Med Rehabil 1997 (in press).

13 Furby A et al. Motor evoked potentials to magnetic stimulation: technical considerations and normative data from 50 subjects. $J$ Neurol 1992; 239: $152-156$.

14 Dietz V, Horstmann G, Berger W. Interlimb co-ordination of leg muscle activation during perturbation of stance in humans. $J$ Neurophysiol 1989; 62: 680-693.

15 Kuhn RA. Functional capacity of the isolated human spinal cord. Brain 1950; 73: $1-51$.

16 Illis LS. Is there a central pattern generator in man? Paraplegia 1995; 33: $239-240$. 
17 Calancie B et al. Voluntary stepping after chronic spinal cord injury. Evidence for a central rhythm generator for locomotion in man. Brain 1994; 117: 1143 - 1159.

18 Bussel B et al. Myoclonus in a patient with spinal cord transection. Possible involvement of the spinal stepping generator. Brain 1988; 111: $1235-1245$.

19 Bussel B, Roby-Brami A, Yakovleff A, Bermis N. Late flexion reflex in paraplegic patients. Evidence for a spinal stepping generator. Brain Res Bull 1989; 22: 53-56.

20 Rossignol S, Barbeau H. New approaches to locomotor rehabilitation in spinal cord injury. Ann Neurol 1995; 37: $555-$ 556.

21 Fung J, Stewart JE, Barbeau H. The combined effects of clonidine and cyproheptadine with interactive training on the modulation of locomotion in spinal cord injured subjects. $J$ Neurol Sci 1990; 100: 85-93.

22 Wernig A, Müller A. Laufband locomotion with body weight support improved walking in persons with severe spinal cord injuries. Paraplegia 1992; 30: 229-238.

23 Wernig A, Müller S, Nanassy A, Cagol E. Laufband therapy based on 'rules of spinal locomotion' is effective in spinal cord injured persons. Eur J Neurosci 1995; 7: 823-829.
24 Harkema SJ et al. Human lumbosacral spinal cord interprets loading during stepping. J Neurophysiol 1997; 77: 797-811.

25 Pearson KG, Collins DF. Reversal of the influence of group Ib afferents from plantaris on activity in medial gastrocnemius muscle during locomotor activity. J Neurophysiol 1993; 70: $1009-1017$.

26 Dietz V, Gollhofer A, Kleiber M, Trippel M. Regulation of bipedal stance: Dependency on load receptors. Exp Brain Res 1992; 89: 229-231.

27 Katho S, El Masry WS. Neurological recovery after conservative treatment of cervical cord injuries. J Bone Joint Surg ( $\mathrm{Br}$ ) 1994; 76-B: $225-228$

28 Curt A, Dietz V. Traumatic cervical spinal cord injury. Relation between somatosensory evoked potentials, neurological deficit, and hand function. Arch Phys Med Rehabil 1996; 77: 48 - 53.

29 Bregman BS et al. Recovery from spinal cord injury mediated by antibodies to neurite growth inhibitors. Nature 1995; 378: $498-$ 499. 